\title{
ON THE LOCATION OF THE SINGULARITIES OF THE FUNCTION GENERATED BY THE BERGMAN OPERATOR OF THE SECOND KIND
}

\author{
PAUL ROSENTHAL
}

\begin{abstract}
Let $g(x, y)=P_{2}(f)$ be Bergman's operator of the second kind, $f(q)$ analytic at $q=0$. The purpose of this paper is to generalize a previous result of the author on the location of the singularities of $g(x, y)$ when $f(q)$ had only a simple pole. $f(q)$ now is assumed to be a rational function whose poles are distributed along the arc of a circle. An order relation is also obtained for $g(x, y)$ for certain fixed $x$ and $y$ sufficiently large and positive.
\end{abstract}

In Cartesian coordinates the differential equation (see below) becomes $\psi_{x x}+\psi_{y y}-(1 / 3 x) \psi_{x}=0$, abbreviated GASPT; see reference [10]. An integral operator approach to this equation, and an investigation of its singularities was pioneered by Gilbert [11], [12]. It should be noted, however, that the operator used by Gilbert and that used by the author are not the same. Consequently, the results of this paper are for a different class of solutions than investigated by Gilbert.

$P_{2}(f)$ maps functions $f(q)$ analytic at $q=0$ into solutions of the partial differential equation (a case arising in fluid flow analysis) $g_{z z^{*}}+$ $N\left(\left(z+z^{*}\right) / 2\right)\left(g_{z}+g_{z^{*}}\right)=0, \quad z=x+i y, z^{*}=x-i y$,

$$
N(x)=\left(1+b_{1}(-x)^{2 / 3}+\cdots\right) /-12 x
$$

is analytic for $-\infty<x<0$. As in [9], we assume $N(x)=1 /-12 x$ (Tricomi case) and $z^{*}=x-i y=\bar{z}$ (conjugate of $z$ ). With these assumptions,

$$
\begin{gathered}
g(x, y)=\int_{c} E(u) f(q)\left(1-t^{2}\right)^{-1 / 2} d t, \quad u=t^{2} z / 2 x, \quad q=\frac{1}{2} z\left(1-t^{2}\right), \\
E(u)=u^{-1 / 6} F\left(\frac{1}{6}, \frac{2}{3}, \frac{1}{3} ; \frac{1}{u}\right) \quad \text { or } \quad u^{-5 / 6} F\left(\frac{5}{6}, \frac{4}{3}, \frac{5}{3} ; \frac{1}{u}\right),
\end{gathered}
$$

Presented to the Society, November 16, 1972; received by the editors December 19, 1972 and, in revised form, August 30, 1973.

AMS (MOS) subject classifications (1970). Primary 35C15, 35J15; Secondary 30A10, 30A68, 33A30, 44A15.

Key words and phrases. Bergman operator of the second kind, hypergeometric function, singular points.

(c) American Mathematical Society 1974 
$F$ is the hypergeometric function (we only treat the first case for $E(u)$ since the second case parallels the first case [9]), $c$ (the path of integration) is the semicircle $t=e^{i a}, 0 \leqq a \leqq \pi$, all integrals are in the improper (complex) Riemann sense, $W=\left\{(x, y)\left|3^{1 / 2}\right| x \mid<y, x \leqq 0, y>0\right\}$, our basic domain [2, p. 107].

THEOREM 1. Let $f(q)$ be the rational function

$$
\left(a_{m} q^{m}+\cdots+a_{0}\right) /\left(b_{p} q^{p}+\cdots+b_{0}\right), \quad p>m
$$

(when $p=1, f(q)$ reduces to the case treated in [9]) whose poles $q=A_{j}$ satisfy the conditions $x_{j}+i y_{j}=A_{j} \in W,\left|A_{j}\right|=M,\left|2 x_{j}\right|>\max \left|x_{j}\right|,\left|x_{j}\right|>0,1 \leqq j \leqq n$. Let $R=\left\{(x, y)\left|(x, y) \in W, \min \frac{2}{3}\right| x_{j}|\leqq| x \mid<M, 0<y<\min 2 y_{j}, 1 \leqq j \leqq n\right\}$, $S=\left\{(x, y)\left|(x, y) \in W, \min \frac{2}{3}\right| x_{j} \mid>x>0,1 \leqq j \leqq n\right\}, D=(R \cup S) \subset W$. Then, (1) for all $(x, y) \in D, g(x, y)$ is analytic, and (2) $\left(2 x_{j}, 2 y_{j}\right),\left(\frac{2}{3} x_{j}, 2 y_{j}\right)$ are singular points of $g(x, y)$ defined by $(1)$.

THEOREM 2. Let $Q$ be compact, nonempty, and $\subset S_{1}=\{x|0<| x \mid<$ $\min \frac{2}{3}\left|x_{j}\right|, x$ negative, $\left.1 \leqq j \leqq n\right\}$. Let $h(y)=g(x, y), x \in Q,(x, y) \in W$. Then $h(y)=o(1)$ (small o of 1) as $y \rightarrow \infty$ uniformly in $x \in Q$.

Proof of Theorem 1. (Some of our methods of proof are based on those in [9].) We write

$$
f(q)=\sum_{j=1}^{n} f_{j}(q), \quad f_{j}(q)=\sum_{k=1}^{s \geqq 1} g_{k}(q), \quad g_{k}(q)=b_{k}\left(q-A_{j}\right)^{-k}
$$

(the partial fraction expansion of $f(q)$ ), and $s$ is the order of the pole at $q=A_{j}$. Consider the general term $g_{k}(q)$. Using the series definition for $F$, expanding $g_{k}(q)$ in a Taylor series about $q=0$, and then using the formula [1, p. 33]

$$
\int_{c} t^{-1 / 3}\left(1-t^{2}\right)^{n+1 / 6} \frac{d t}{\left(1-t^{2}\right)^{1 / 2}}=-\left(1-e^{2 \pi i / 3}\right) \Gamma\left(\frac{1}{3}\right) \frac{\Gamma\left(n+\frac{2}{3}\right)}{\Gamma(n+1)}, \quad n>-2 / 3,
$$

$\Gamma$ is the Gamma function, we obtain from (1)

$$
\begin{gathered}
g_{j}(x, y)=\left(\frac{2 x}{x+i y}\right)^{1 / 6} \\
\left(f_{j}^{(1)}(x, y)=\sum_{p=0}^{\infty} a_{1}\left(p, s, \frac{x}{A_{j}}\right)\left(\frac{z}{2 A_{j}}\right)^{p}+f_{j}^{(2)}(x, y)=\sum_{p=1}^{\infty} a_{2}\left(p, s, \frac{x}{A_{j}}\right)\left(\frac{2 x}{z}\right)^{p}\right),
\end{gathered}
$$

where

$$
a_{1}=\sum_{k=1}^{s} B_{k} \frac{\Gamma\left(p+k-\frac{1}{2}\right)}{\Gamma\left(p+\frac{5}{6}\right)} F\left(\frac{1}{6}, p+k-\frac{1}{2}, \frac{1}{3} ; \frac{x}{A_{j}}\right) \text {, }
$$


$B_{k}$ are constants, $p \geqq 0$ (when $s=1$, we obtain the case treated in [9]), $a_{2}\left(p, s, x / A_{j}\right)$ is analytic in $x$ ( $x$ extended to complex values) for $|x|<$ $\left|A_{j}\right|, p \geqq 1$, and is uniformly bounded for all $|x| A_{j} \mid \leqq 1-d$ and for all $p \geqq 1,1>d>0$, and arbitrarily small. All limit operations used to derive (2) are permissible providing $|z| \leqq\left|A_{j}\right|-d,|x| \leqq 2^{-1}\left|A_{j}\right|-d, \quad 1-d \geqq$ $2|x| /|z|, d>0$ and sufficiently small.

Using next the formula [7, p. 240]

$$
\begin{aligned}
& \frac{\Gamma(c-a) \Gamma(a)}{\Gamma(c)} F(a, b, c ; w) \\
& =\frac{\Gamma(1+b-c)}{\Gamma(1+b-a)} \Gamma(c-a) w^{a-c}(1-w)^{c-a-b} F\left(c-a, 1-a, 1+b-a ; w^{-1}\right) \\
& \quad+\frac{\Gamma(1+b-c) \Gamma(a)}{\Gamma(1+a+b-c)} e^{i \pi a} w^{-a} F\left(a, 1+a-c, 1+a+b-c ; 1-w^{-1}\right),
\end{aligned}
$$

$\operatorname{Im} w>0$, we can write $f_{j}^{(1)}$ of (2) as

$$
\begin{aligned}
f_{j}^{(1)}= & \left(h_{j}^{(1)}(x, y)=\sum_{p=0}^{\infty} c_{j}^{(1)}\left(p, \frac{x}{A_{j}}\right) z_{1}^{p}\right) \\
& +\left(h_{j}^{(2)}(x, y)=\sum_{p=0}^{\infty} c_{j}^{(2)}\left(p, \frac{x}{A_{j}}\right) z_{2}^{p}\right),
\end{aligned}
$$

$z_{1}=z / 2 A_{j}, z_{2}=z / 2\left(A_{j}-x\right)$, where the $c_{j}^{(k)}$ are finite linear combinations of hypergeometric functions whose coefficients are ratios of Gamma functions, $k=1,2$. We now consider the defining series for $h_{j}^{(k)}, k=1,2$, of (3) (for convergence (uniform), analyticity, and analytic continuation with respect to $x$ and $y$ ). Let $y$ be extended to complex values. Fix $x$ in $Q_{1}=\left\{x|0<d \leqq| x|\leqq| A_{j} \mid-d, d\right.$ sufficiently small, $7 \pi / 6 \geqq \arg x \geqq 5 \pi / 6, x$ complex\}. From an estimate of the remainder term for $F$ when we consider the behavior of $F$ for large $p$ [7, p. 235] and the asymptotics for the ratio of two Gamma functions [5, p. 47], we obtain

$$
\lim _{p \rightarrow \infty}\left|c_{j}^{(k)}\left(p, x \mid A_{j}\right)\right|^{-1 / p}=1
$$

(the radius of convergence of the Taylor series in (3) when $z_{1}$ and $z_{2}$ are temporarily considered as independent variables), uniformly for $x$ in $Q_{1}, k=1,2$,

$$
\left|\arg \left(c_{j}^{(k)}\left(p, x / A_{j}\right)\right)\right|<d, \quad \pi / 2>d>0,
$$

$p$ sufficiently large, uniformly for $x$ in $Q_{1}, k=1,2$ (we subtract out nonzero constant arguments),

$$
c_{j}^{(k)}\left(p=x_{1}+i y_{1}, x / A_{j}\right)
$$


is analytic in $p$ in the half plane $x_{1} \geqq 0, x$ in $Q_{1}, k=1,2$. (This is a consequence of the analyticity of the Gamma function and the hypergeometric function in its parameters.)

$$
\left|c_{j}^{(k)}\left(p=r e^{i a}, x \mid A_{j}\right)\right|<e^{d r},
$$

$d>0$ and arbitrarily small, $r>0$ and sufficiently large, uniformly for $|a| \leqq \pi / 2$ and $x$ in $Q_{1}, k=1,2$. We obtain (7) from an estimate for the remainder term for $F$ for large $p$ (complex), $|\arg p| \leqq \pi / 2$ [7, p. 235], noting the definition of the $c_{j}^{(k)}$ in (3), (2), and the formula stated after (2).

LEMMA 1. $\left(2 x_{j}, 2 y_{j}\right)$ and $\left(\frac{2}{3} x_{j}, 2 y_{j}\right)$ are singular points, respectively, of $h_{j}^{(1)}$ and $h_{j}^{(2)}$ of (3).

Proof of Lemma 1. (4) and (5) satisfy the conditions of a theorem of Dienes [5, p. 227], which in turn implies Lemma 1. (Note we are fixing $x$ in $Q_{1}$ and considering $y$ as an independent complex variable.)

LEMMA 2. $\left(2 b x_{j}, 2 b y_{j}\right)$ and $\left(2 c x_{j} /(1+2 c), 2 c y_{j}\right), b, c \geqq 1$ and arbitrary, are the only possible singular points in $W$, respectively, of $h_{j}^{(1)}$ and $h_{j}^{(2)}$ of (3), $x$ in $Q_{1}$.

Proof of Lemma 2. (6) and (7) satisfy the conditions of a theorem of LeRoy and Lindelöf [5, p. 340] which in turn implies $z_{1}=b, z_{2}=c$, $b, c \geqq 1$ are the only possible singular points of the Taylor series in (3). Hence Lemma 2 holds.

LeMma 3. $\left(\frac{2}{3} x_{j}, 2 y_{j}\right)$ is a regular point of $h_{j}^{(1)}$ of (3).

Proof of Lemma 3. Let $x, y$ be independent complex variables. We can then find nonempty disc neighborhoods $N(x), N(y)$ with centers at $x=\frac{2}{3} x_{j}$ and $y=y_{0}, 0<y_{0} \leqq 2 y_{j}$ such that the general term $D_{j}^{(1)}(p, x, y)=$ $c_{j}^{(1)}\left(p, x / A_{j}\right)\left((x+i y) / 2 A_{j}\right)^{p}, p \geqq 0$, of (3) is analytic for $x$ in $N(x)$ and $y$ in $N(y)$ and such that the defining series for $h_{j}^{(1)}, \sum_{p=0}^{\infty} D_{j}^{(1)}(p, x, y)$, converges uniformly on compact sets $Q_{1} \subset N(x), Q_{2} \subset N(y)$ since the asymptotics for $F$ for large $p$ [7, p. 235] and those for the Gamma function $\left[6\right.$, p. 47] imply we can uniformly dominate $\sum_{p=0}^{\infty} D_{j}^{(1)}(p, x, y)$. Hence we conclude $h_{j}^{(1)}$ is analytic at $\left(\frac{2}{3} x_{j}, 2 y_{j}\right)$. This completes the proof of Lemma 3.

LemMa 4. $\left(2 x_{j}, 2 y_{j}\right)$ is a regular point of $h_{j}^{(2)}(x, y)$ of $(3)$.

Proof of Lemma 4. We proceed as in the proof of Lemma 3 and thus conclude $h_{j}^{(2)}(x, y)$ is analytic for $x$ in $N_{1}(x)$ and $y$ in $N_{1}(y)$ with centers respectively at $x=2 x_{j}$ and $y=y_{0}, 0<y_{0}<2 y$. Hence by a theorem of Hartogs (extension theorem) [3, p. 141], we conclude $h_{j}^{(2)}(x, y)$ is analytic at $\left(2 x_{j}, 2 y_{j}\right)$. (Note we are fixing $x$ in $N_{1}(x)$ with center at $2 x_{j}$ and extending 
analytically in $y, h_{j}^{(2)}(x, y)$, this is permissible by a theorem of Leroy and Lindelöf [5, p. 340].) This completes the proof of Lemma 4.

Lemma 5. $g_{j}(x, y)$ of $(2)$ is singular at the points $\left(\frac{2}{3} x_{j}, 2 y_{j}\right),\left(2 x_{j}, 2 y_{j}\right)$, $1 \leqq j \leqq n$.

Proof of Lemma 5. The conditions imposed on the $x_{j}$ in the hypothesis of Theorem 1 imply $\frac{2}{3} x_{j}, 2 x_{j}$ are in the domain of regularity of $a_{2}\left(p, x \mid A_{j}\right), p>1$, of (2). We can find nonempty neighborhoods $N_{2}(x)$ and $N_{2}(y)$ with centers respectively at $x=\frac{2}{3} x_{j}, 2 x_{j}$ and $y=2 y_{j}$ such that $f_{j}^{(2)}(x, y)$ of (2) is analytic at $\left(2 x_{j}, 2 y_{j}\right),\left(\frac{2}{3} x_{j}, 2 y_{j}\right)$ (we majorize the series for $\left.f_{j}^{(2)}\right)$. Lemmas $1,2,3$, and 4 imply the singular points $\left(2 x_{j}, 2 y_{j}\right)$ and $\left(\frac{2}{3} x_{j}, 2 y_{j}\right)$ respectively of $h_{j}^{(1)}(x, y)$ and $h_{j}^{(2)}(x, y)$ are not removed under addition of $h_{j}^{(1)}$ to $h_{j}^{(2)}$ of (3). Hence from (2) we obtain the conclusion of Lemma 5.

Lemma 6. Let $g(x, y)=\sum_{j=1}^{n} g_{j}(x, y)$ (permissible by the linearity of $\left.P_{2}(f)\right),(x, y)$ in $D$ (see Theorem 1 for the definition of $\left.D\right)$. Then $g(x, y)$ is analytic for all $(x, y)$ in $D$ and is singular at the points $\left(\frac{2}{3} x_{j}, 2 y_{j}\right),\left(2 x_{j}, 2 y_{j}\right)$, $1 \leqq j \leqq n$.

Proof of Lemma 6. Consider the term $g_{j_{0}}(x, y), j_{0}$ fixed, $1 \leqq j_{0} \leqq n$. The conditions imposed on the $x_{j}$ in Theorem 1 imply $\frac{2}{3} x_{j}, 2 x_{j}, 1 \leqq j \leqq n$, are in the domain of regularity of $a_{2}\left(p, x / A_{j}\right)$ of (2), $p \geqq 1$. Consider the finite sequence $g_{j}(x, y), 1 \leqq j \leqq n, j \neq j_{0}$. The methods of proof in Lemmas 3,4 , and 5 and the conditions imposed on the $x_{j}$ in the hypothesis of Theorem 1 imply $\left(\frac{2}{3} x_{j_{0}}, 2 y_{j_{0}}\right),\left(2 x_{j_{0}}, 2 y_{j_{0}}\right)$ are regular points of $g_{j}(x, y)$, $1 \leqq j \leqq n, j \neq j_{0}$. Hence the finite sum $\sum_{j=1 ; j \neq j_{0}}^{n} g_{j}(x, y)$ is analytic at $\left(\frac{2}{3} x_{j_{0}}, 2 y_{j_{0}}\right),\left(2 x_{j_{0}}, 2 y_{j_{0}}\right)$. Lemma 5 then implies the second part of Lemma 6 . Let

$$
\begin{aligned}
R_{j} & =\left\{(x, y) \mid(x, y) \text { in } W, \frac{2}{3}\left|x_{j}\right| \leqq|x|<\left|A_{j}\right|, 0<y<2 y_{j}\right\}, \\
S_{j} & =\left\{(x, y) \mid(x, y) \text { in } W, \frac{2}{3}\left|x_{j}\right|>|x|>0\right\}, \quad D_{j}=R_{j} \cup S_{j}, \\
T_{j} & =\left\{(x, y) \mid(x, y) \text { in } W, 0<|x|<\left|A_{j}\right|=M\right\} .
\end{aligned}
$$

Then, as in the proof of Lemma $5, f_{j}^{(2)}(x, y)$ of (2) is analytic for all $(x, y)$ in $T_{j}, D \subset T_{j}$. Proceeding then as in the proofs of Lemmas 3, 4, and 5 , we conclude $g_{j}(x, y)$ is analytic for all $(x, y)$ in $D_{j}, 1 \leqq j \leqq n$. Since $D \subset \bigcap_{j=1}^{n} D_{j}$, we conclude the first part of Lemma 6.

Since Lemma 6 is a statement of Theorem 1, this completes the proof of Theorem 1.

Proof of Theorem 2. Let $x$ be fixed and in $Q$ of Theorem 2. Let $g_{j}(x, y)$ be the general term in the hypothesis of Lemma 6. (5) and (6) in the proof of Theorem 1 imply $h_{j}^{(k)}(x, y), k=1,2$, of (3) satisfy the 
conditions of a theorem of Lindelöf [5, p. 343]. This theorem implies $h_{j}^{(k)}(x, y) \rightarrow 0$ as $y \rightarrow \infty$ uniformly for $x$ in $Q,(x, y)$ in $W$. (Note all $x$ in $Q$ are in the domain of regularity of $a_{2}\left(p, x \mid A_{j}\right), p \geqq 1$, of (2).) Hence $f_{j}^{(2)}(x, y)$ (of (2)) $\rightarrow 0$ as $y \rightarrow \infty$, uniformly for $x$ in $Q,(x, y)$ in $W(2)$ and the definition of $g(x, y)$ as the finite sum $\sum_{j=1}^{n} g_{j}(x, y),(x, y)$ in $D$ (see Lemma 6 of Theorem 1), then imply Theorem 2.

We note in closing, D. Colton and R. Gilbert in [4] give necessary and sufficient conditions for a point $z$ to be a singular point of Bergman's operator of the first kind (see [2]). However, our problem of mapping rational functions into solutions of the above partial differential equation and then investigating the singularities of the mapped solution seems to have not been treated via the Bergman operator of the second kind.

\section{REFERENCES}

1. S. Bergman, On an initial value problem in the theory of two-dimensional transonic flow patterns, Pacific J. Math. 32 (1970), 29-46. MR 40 \#8332.

2. - Integral operators in the theory of linear partial differential equations, 2 nd rev. ed., Ergebnisse der Math. und ihrer Grenzgebiete, Band 23, Springer-Verlag, New York, 1969. MR 39 \#596.

3. S. Bochner and W. Martin, Several complex variables, Princeton Math. Series, vol. 10, Princeton Univ. Press, Princeton, N.J., 1948. MR 10, 366.

4. D. Colton and R. Gilbert, Singularities of solutions to elliptic partial differential equations with analytic coefficients, Quart. J. Math. Oxford Ser. (2) 19 (1968), 391-396. MR 38 \#6206.

5. P. Dienes, The Taylor series, Oxford Univ. Press, 1931.

6. A. Erdélyi, et al., Higher transcendental functions. Vol. I. The Hypergeometric function, Legendre functions, McGraw-Hill, New York, 1953. MR 15, 419.

7. Y. Luke, The special functions and their approximations. Vol. I, Math. in Science and Engineering, vol. 53, Academic Press, New York, 1969. MR 39 \#3039.

8. T. M. MacRobert, On an asymptotic expansion of the hypergeometric function, Proc. Edinburgh Math. Soc. vol. 1, 42 (1923), 84-87.

9. P. Rosenthal, On the singularities of the functions generated by the Bergman operator of the second kind, Pacific J. Math. (to appear).

10. A. Weinstein, Generalized axially symmetric potential theory, Bull. Amer. Math. Soc. 59 (1953), 20-38. MR 14, 749.

11. R. Gilbert, On the singularities of generalized axially symmetric potentials, Arch. Rational Mech. Anal. 6 (1960), 171-176. MR 22 \#11231.

12. - Function theoretic methods in partial differential equations, Math. in Science and Engineering, vol. 54, Academic Press, New York, 1969. MR 39 \#3127.

\footnotetext{
Department of Mathematics, Stanford University, Stanford, California 94305
}

Current address: Apt. 103, 90 Heather Avenue, San Francisco, California 94118 